\title{
TEORÍA DEL CONTROL
}

\author{
Luis Arturo Polania Q. \\ Esp. en Matemática Avanzada, Universidad \\ Nacional de Colombia, Mag. en Ciencias \\ Matemáticas. Universidad Nacional de Colombia, \\ Profesor T.C. Universidad Surcolombiana
}

El hombre ha girado, en buena parte, alrededor de la idea de controlar a lo largo de toda su historia. Es probable, que mucho antes de la aparición de la historia escrita ya se conocían rudimentarios mecanismos de control (de riego, por ejemplo). Se investiga y se experimenta con el anhelo de conocer y así poder establecer medios para controlar procesos de la más variada naturaleza.

La invención del regulador automático de velocidad de las máquinas a vapor de James Watt, hacia mediados del siglo XVIII, es citado como la primera aparición del control automático.

En este siglo, durante los años veinte y treinta, hubo importantes aportes en áreas como la teoría de circuitos, la electrónica y el control de procesos químicos. En 1941 L. Hazen publicó un libro titulado "Theory of Servomechanism", obra que es considerada como uno de los primeros esfuerzos serios por el desarrollo de una teoría general del control automático. Esta publicación se considera fundamental, por el establecimiento de modelos matemáticos y analogías de los procesos de control.

El establecimiento definitivo del control como ciencia se sitúa durante la segunda guerra mundial. Resultado obvio del esfuerzo por desarrollar nuevos y precisos mecanismos que asegurasen la superioridad técnica. Las características dinámicas de los dispositivos de alta efectividad propuestos en ese entonces, hicieron necesaria la creación de una teoría completamente nueva.

Entre los años de 1945 y 1950 se consolidaron los avances recién logrados. Luego viene la apertura a nuevas áreas de aplicación: como la economía, la biología y la navegación aero-espacial entre otros. No se puede dejar a un lado, la entrada en escena de los computadores y de las técnicas de simulación. Hasta el punto que hoy en día no se puede concebir estudiar o investigar en el área del control sin la ayuda de adecuadas técnicas de simulación.

\section{Sistemas de Control}

Uno de los aspectos más atractivos de la teoría del control es su aplicabilidad a procesos de tipo general. Entenderemos por un proceso algún movimiento o acción. que tenga lugar a medida que el tiempo transcurre. En general consideramos un sistema, cuyo estado se describe por un punto en un espacio llamado espacio de fase.

Sobre el sistema se formulará una estructura que permitirá, una vez se especifique una "política de control", determinar el curso posterior del proceso en base a, conocimiento del estado previo del sistema. Dicha estructura será llamada la dinámica. Una política de control, o simplemente un control que determina el 
comportamiento dinámico pertenecerá a un conjunto dado de funciones del tiempo.

Es preciso insistir en que, a cada punto del espacio de fase corresponda una "trayectoria" bien determinada en dicho espacio, para cada control admisible.

\section{Elementos esenciales que aparecen en un problema general de control de un sistema regido por ecuaciones diferenciales ordinarias}

(i) Sistema S (que puede ser un proceso mecánico, proceso eléctrico, proceso químico, proceso industrial, proceso social, etc.). Cuya operación y funcionamiento depende de un cierto número de controles $\left(u_{1}, \ldots, u_{k}\right)$ o parámetros variables dentro de ciertos límites.

(ii) Ley del proceso. Esta ley relaciona el estado, respuesta o salida (output) $x(1)$, una variable $n$-dimensional con el control o entrada (input) $u(t)$, una variable $\mathrm{m}$-dimensional. La ley viene dada por una ecuación (vectorial) diferencial ordinaria, de la forma:

donde $\mathrm{f}$ es una función de $\mathrm{IR} \times \mathrm{IR}^{\mathrm{n}} \times \mathrm{IR}^{\mathrm{m}}$ " a IR .

Esta ecuación puede ser lineal o no. El objetivo final consiste en controlar el proceso mediante un control de ciclo cerrado y de modo óptimo.

(iii) Estado inicial y estado final o conjunto meta. Se señala un estado de partida mediante un valor inicial del estado del sistema $x_{o}=x\left(t_{o}\right)$ en el instante $t_{0}$.

Los diferentes componentes del estado $\mathbf{x}(\mathbf{t})$ que se pretende controlar pueden ser magnitudes tales como posición, velocidad, aceleración, velocidad angular, temperatura, intensidad de corriente, etc. El conjunto meta $G(t)$ es un conjunto dado del espacio IIVde estados que varia con el tiempo de modo continuo. Se trata de llevar el proceso del estado $\mathrm{x}_{\mathrm{o}} \mathrm{a}$ un estado $x\left(t_{1}\right)=\in G\left(t_{1}\right)$.

(iv) La clase de controles. Consideramos funciones continuas a trozos, definidas sobre intervalos de IR con valores en $\mathrm{IR}^{\mathrm{m}}$. Estas serán nuestras funciones de control. De entre ellas llamaremos controles admisibles a aquellas que realizan nuestro objetivo fundamental, es decir, funciones continuas a trozos $u:\left[t_{0}, t_{1}\right] \rightarrow I^{m}$ para algún

$t_{1} \geq t_{o}$ tales que existe una solución

$x(t) d e \frac{\mathrm{dx}}{\mathrm{dt}}=f(t, x(t), u(t)) q u e$

verifica $x\left(t_{o}\right)=x_{o^{\prime}} x\left(t_{f}\right) \in G\left(t_{P}\right)$. De estos debemos distinguir aún el control o controles óptimos.

(v) El índice de funcionamiento o funcional de costo. El funcionamiento del sistema bajo la acción de diferentes controles es, naturalmente, distinto. Su calidad se ha de medir mediante un criterio que consiste en adoptar un costo dado por un funcional dependiente de u de la forma.

$$
J(U)=\int_{t_{0}}^{4} f^{*}(t, x(t), u(t)) d t
$$

siendo $f^{*}$ una función escalar. Si por ejemplo, $f^{*}$ es idénticamente 1 , resulta

$$
J(\mathrm{u})=t_{\mathrm{i}}-t_{\mathrm{o}}
$$




\section{Ejemplo 1}

Considérese un tren de masa $m$ que pasa por la posición $A$ con una velocidad $v$ y ha de ser llevado en línea recta a una posición de reposo en $B$.

Supóngase que está dotado de dos motores iguales que actúan en sentidos contrarios y que son utilizados tanto para acelerar como para frenar.

En este caso, podemos suponer que el tren se mueve siguiendo la segunda Ley de Newton (o sea, la segunda Ley de Newton nos permite formular la dinámica del sistema) :

$$
u(t)=m \frac{d^{2} x(t)}{d t^{2}},
$$

donde $u(t)$ es la fuerza aplicada en el instante $t$, positiva si es de marcha y negativa si es de frenado, $x(t)$ es la distancia en el instante $t$ de A a la posición actual del tren en el sentido de A a B.

El control es $u(t)$, que está a nuestra disposición en cada instante $t$.

El estado inicial es la posición A con velocidad $v$ y el estado final o meta es la posición $\mathrm{B}$ con velocidad $\mathrm{v}=0$. El índice de funcionamiento es el tiempo $\mathrm{T}$ que tarda el tren desde A hasta B aplicando el control $u(t)$, y se desea escoger este control de tal manera que t sea mínimo.

El índice de funcionamiento $J$ es, como se ve, una función del control $u(t)$, que es, a su vez, una función del tiempo. Es pues. un funcional que se suele llamar funcional de costo. Viene a medir la calidad, de acuerdo con un criterio prefijado, del control que apliquemos.
En el caso del tren el funcionamiento el sistema está regido por una sencilla ecuación diferencial ordinaria.

\section{Modelo general de un sistema de control a tiempo continuo y de dimension finita}

Puede ser descrito por :

$$
\begin{aligned}
& \frac{\mathrm{dx}}{\mathrm{dt}}=f(t, x, u, d) \\
& \mathrm{y}=\mathrm{G}(\mathrm{t}, x, u, d) \\
& \mathrm{z}=\mathrm{H}(t, x, u, d)
\end{aligned}
$$

donde $u$ y $d$ son entradas (control y disturbio, respectivamente)

y y z salidas (salida controlada y medida, respectivamente)

t el tiempo

$\mathrm{x}$ es el vector de estado del proceso

Suponiendo la existencia y unicidad de las soluciones globales de la ecuación diferencial, el conocimiento del instante inicial $t_{o}$, del estado inicial $x\left(t_{0}\right)$, y de las entradas presentes y futuras (esto es, $u(t)$ $y d(t)$ para $t_{\mathrm{o}} \leq t<+{ }^{\infty}$ ) permite determinar los valores de $x(t)$, y $(t)$ y $z(t)$ en el presente y en el futuro (esto es, para $\left.\mathrm{t}_{\mathrm{o}} \leq t<+{ }^{\infty}\right)$. Nótese que $t$ es una variable real y $x, u, d$, y y $z$ son funciones del tiempo, con valores en espacios vectoriales de dimensión finita.

NOTA. Se pueden definir procesos regidos por ecuaciones diferenciales z rrciales o por ecuaciones diferenciales con retardos. En este caso precisaríamos de variables de estado definidas en espacios de dimensión infinita.
$M$ 
Para considerar controladores usando microprocesadores precisaremos de procesos para los cuales el tiempo es una variable discreta, definida sobre el conjunto de los números enteros. En este caso las ecuaciones diferenciales serán remplazadas por ecuaciones a diferencias. Por ejemplo,

$$
\begin{aligned}
& x(\mathrm{k}+\mathrm{I})=\mathrm{F}(t, x(k), u(k), d(k)) \\
& \mathrm{y}(\mathrm{k})=\mathrm{G}(t, x(k), u(k), d(k)) \\
& z(\mathrm{k})=\mathrm{H}(t, x(k), u(k), d(k))
\end{aligned}
$$

De nuevo, el conocimiento del instante inicial $t_{0}$, del estado inicial $\mathrm{x}\left(t_{\mathrm{o}}\right)$, y de las entradas presentes y futuras (esto es, $u(k)$ y $d(k)$ para $\left.t_{0} \leq t<+\infty\right)$ permite determinar los valores de $x(k), y(k)$ y $z(k)$ en el presente y en el futuro (esto es, para $\left.\mathrm{t}_{\mathrm{o}} \leq t<+\infty\right)$.

Ejemplo 2

Posicionamiento de una antena parabólica para microondas.

Consideremos una antena parabólica para microondas, colocada en lo alto de un terreno. Se trata de alterar su posición angular con el objeto de contactar estaciones diferentes. La antena debe ser controlada $M$ a distancia, a partir de un laboratorio, el cual exige un atuador local (motor eléctrico que mueve la antena en torno de su eje). También es necesario tener información sobre la posición real de la antena: para ello se usan sensores de posición (un potenciómetro eléctrico ligado al eje de la antena) o sensores de velocidad de rotación (tacogeneradores, por ejemplo).

¿Qué queremos hacer con la antena? primero, especificar el ángulo para que la antena apunte a determinada emisora. deseamos que ella obedezca el comando.
Esto es, marcando un ángulo con un botón en el laboratorio, la antena en lo alto del terreno, gira hasta el ángulo indicado. $O$ más modernamente, escogiendo un ángulo en un programa de control en el computador del laboratorio, la antena gira hasta el ángulo indicado. Así mismo, debe mantenerse el mismo ángulo sobre la acción de los vientos normales de la región. Más aún, para no dañar los sensores. conviene que la antena, al moverse de una posición para otra, no sobrepase la posición de llegada.

Hasta aquí se ha definido un problema de ingeniería, mas no un problema de matemática.

El primer trabajo del ingeniero consiste en escoger atuadores y sensores. En el caso, calcular la potencia necesaria para el motor (depende de la inercia de la antena y de la fuerza máxima de los vientos) y escoger motor y sensores entre los disponibles en el mercado, considerando costos y durabilidad. Terminada la escogencia, el ingeniero pasa a proyectar el controlador, un equipamiento que, a partir de las medidas de los sensores y del ángulo deseado, haga que el motor se mueva debidamente. ¿Qué tipo de equipamiento será ese ? Un motor eléctrico posee dos entradas eléctricas: La que alimenta el campo electromagnético generado por las bobinas de campo, fijas, y la que alimenta el campo magnético generado por el rotor o armadura. La interacción de estos dos campos hace girar el motor. Normalmente, se deja fija una de las entradas y se varia la otra, ya que el torque del motor es proporcional al producto de las corrientes en las bobinas de campo y armadura. La entrada variable puede ser usada para controlar el motor, y de ahí la antena, haciéndola girar más rápidamente o más lentamente. o invirtiendo el sentido del movimiento. 
El controlador deberá ser un circuito eléctrico que, recibiendo las señales eléctricas viniendo de los sensores y la señal eléctrica viniendo del botón de control en el laboratorio, genera la señal eléctrica que controla el motor.

Así entonces, escogido el equipamiento, el ingeniero formaliza el problema. Para eso deberá utilizar diferentes modelos matemáticos para el sistema motor - antenasensores, conduciendo a problemas matemáticos diferentes, de acuerdo con el modelo escogido. Un modelo matèmático para el proceso motor - antena - sensores, es aquel donde las funciones $\mathrm{F}, \mathrm{G}$ y $\mathrm{H}$ son lineales, teniendo la forma :

$$
\begin{aligned}
& \frac{d x}{d t}=A x+B u+E d, \\
& y=C x+D u+K d \\
& z=C_{1} x+D_{1} u+K_{1} d
\end{aligned}
$$

donde A, B, C, D, K, C $, D_{1}, K_{1}$, son matrices constantes con dimensiones convenientes.

\section{REFERENCIAS BIBLIOGRÁFICAS}

1. Azevedo Da Silveira, M; Controle De Sistemas Lineares, IMPA 24 - 28 Julio, 1995.

2. Tocancipá, Alfonso: Teoría del Control, Boletín de Matemáticas. Volumen X. 1980. 